\title{
Correção cirúrgica do aneurisma de ventrículo esquerdo: comparação entre as técnicas de sutura linear e reconstrução geométrica
}

Cássio José SGARBI* , Roberto Vito ARDITO*, Rinaldo Costa SANTOS*, Renata Andrea B. BOGDAN*$^{*}$, Franscismar Vidal de ARRUDA JUNIOR*, Elaine Moraes da SILVA**, Ariane Cristina M. BENITES**, Wilma R. ARDITO***, Maria de Fátima F. B.NEVES***

RBCCV 44205-515

Sgarbi C J, Ardito R V, Santos R C, Bogdan R A B, Arruda Junior F V, Silva E M, Benites A C M, Ardito W R, Neves M F F B - Correção cirúrgica de aneurisma de ventrículo esquerdo: comparação entre as técnicas de sutura linear e reconstrução geométrica. Rev Bras Cir Cardiovasc 2000; 15 (4): 293-301.

RESUMO: Objetivos: Avaliar a evolução até 15 anos de acompanhamento dos pacientes submetidos a correção cirúrgica do aneurisma de ventrículo esquerdo, comparando as técnicas de sutura linear $(R L)$ e reconstrução geométrica(RG).

Casuística e Métodos: Foram estudados 213 pacientes; destes, $166(77,9 \%)$ eram do sexo masculino. A idade média foi de 53,1 anos ( $\mathrm{DP}=9,9$ anos), variando de 24 a 73 anos. Do total dos pacientes, 145 (68\%) foram operados pela técnica de SL e $68(32 \%)$ submetidos a RG de VE. A sobrevivência tardia foi medida com o auxílio do método de Kaplan - Meier. Também foram avaliadas a presença de trombo mural, a incidência de óbitos intra-hospitalares e a possibilidade de revascularização concomitante do miocárdio. As diferenças estatísticas ( $p$ valor) foram medidas pelos métodos de "Log Rank" nas curvas atuariais de sobrevivência e pelo método de "Nonparametric Test" (teste de duas simples proporções) nas demais comparações.

Resultados: O estudo atuarial após 10 anos de acompanhamento dos pacientes submetidos a SL e RG do VE revelou uma sobrevivência de $47,19 \%(E P=0,056)$ e de $63,55 \%(E P=0,068)$, respectivamente, não apresentando diferença significativa $(p=0,56)$. Nos dois grupos associados, a sobrevivência foi de $51,34 \%$ $(E P=0,0473)$ com 10 anos e de 35,77\% (EP=0,0648) com 15 anos de acompanhamento. A incidência de óbitos intra-hospitalares foi de $9,5 \%$ no primeiro grupo e de $16,6 \%$ no segundo, com $p=0,17$ e a retirada de trombos da cavidade ventricular esquerda foi de $31,72 \%$ e $44,12 \%$, respectivamente, com $p=0,07$. Foram revascularizados $69 \%$ dos pacientes submetidos a SL e $85,3 \%$ dos submetidos a RG de VE. Demonstrou-se uma sobrevivência superior, em 10 anos de acompanhamento, para os pacientes revascularizados $(p=0,008)$.

Conclusões: Não houve diferença significativa na curva de sobrevivência dos pacientes comparando as técnicas de sutura linear e reconstrução geométrica de VE. Também foi demonstrado a superioridade na evolução dos pacientes revascularizados.

DESCRITORES: Ventrículo cardíaco, cirurgia. Aneurismas cardíacos, cirurgia. Aneurismas de ventriculo, cirurgia, métodos. Retalhos cirúrgicos. Anastomoses cirúrgicas, métodos. Ventrículo cardíaco, cirurgia, estudo comparativo.

Trabalho realizado no Instituto de Moléstias Cardiovasculares (IMC) de São José do Rio Preto. São José do Rio Preto, SP, Brasil. Apresentado ao $27^{\circ}$ Congresso Nacional de Cirurgia Cardíaca. Rio de Janeiro, RJ. 23 a 25 de março de 2000.

* Do Departamento de Cirurgia Cardíaca do Instituto de Moléstias Cardiovasculares de São José do Rio Preto, SP, Brasil.

** Do Departamento de Anestesiologia do I.M.C. de São José do Rio Preto.

*** Do Departamento de Terapia Intensiva do I.M.C. de São José do Rio Preto.

Endereço para correspondência: Cássio José Sgarbi. Av. Santos Dumont, 2200. Santa Maria. Uberaba, MG, Brasil. CEP: 38050.400. Tel: (34) 33149485. e-mail: c.sgarbi@uol.com.br 
Sgarbi C J, Ardito R V, Santos R C, Bogdan R A B, Arruda Junior F V, Silva E M, Benites A C M, Ardito W R, Neves M F F B - Correção cirúrgica de aneurisma de ventrículo esquerdo: comparação entre as técnicas de sutura linear e reconstrução geométrica. Rev Bras Cir Cardiovasc 2000; 15(4): 293-301.

\section{INTRODUÇÃO}

O aneurisma de ventrículo esquerdo (VE) é uma das complicações do infarto agudo do miocárdio. $O$ progressivo aumento da cavidade ventricular resulta em um proporcional aumento da tensão na parede ventricular e da pressão intracavitária, diminuindo a habilidade do restante da musculatura viável para contrair, aumentando a demanda de oxigênio do miocárdio. Insuficiência cardíaca congestiva (1) e angina de peito são comumente encontrados, além de arritmias, tromboembolismo e outros.

É encontrado em $10 \%$ dos sobreviventes do infarto agudo do miocárdio (2) e é quatro vezes mais freqüente em infartos que afetam a parede anterior do que a inferior (3).

O tratamento cirúrgico do aneurisma de VE foi iniciado por LIKOF \& BAILEY(4) quando foi feita a exclusão cirúrgica do mesmo sem abrir a cavidade ventricular. Reparo linear do aneurisma do VE, com ressecção e sutura, utilizando circulação extracorpórea, foi proposto por COOLEY et al. (5), em 1958. Essa técnica operatória foi utilizada de forma inalterada, por mais de duas décadas, de seu trabalho inicial, apresentando resultados satisfatórios.

Após fundamentado o conceito de correção linear do aneurisma do VE, as atenções se voltaram para a procura de novos métodos de reconstrução para restaurar a geometria do VE. Este conceito foi introduzido por JATENE (1), em 1985, e mais tarde modificado por DOR et al. (6). Em 1989, após três décadas, COOLEY ${ }^{(7)}$ propôs o reparo intracavitário ou endoaneurismorrafia.

Para correção cirúrgica do aneurisma de VE tipo reconstrução geométrica, BRAILE et al. (8) desenvolveram uma prótese semi-rígida de pericárdio bovino, sendo esta utilizada na maioria dos nossos pacientes.

O presente estudo tem como objetivo avaliar a evolução no intervalo de 15 anos de acompanhamento dos pacientes submetidos a correção cirúrgica do aneurisma de ventrículo esquerdo, comparando as técnicas de reparo linear e reconstrução geométrica.

\section{CASUÍSTICA E MÉTODOS}

De janeiro de 1973 a outubro de 1996, 213 pacientes foram submetidos a operação para correção do aneurisma de ventrículo esquerdo. Destes, $166(77,9 \%)$ eram do sexo masculino. A idade média foi de 53,1 anos (desvio padrão de 9,9 anos), variando de 24 a 73 anos.
Do total do pacientes, 145 (68\%) foram operados pela técnica de sutura linear e $68(32 \%)$ submetidos a reconstrução geométrica do VE. Foram excluídos do estudo os pacientes que apresentavam doenças valvular reumática ou congênita.

Foram avaliadas a presença de trombo mural, óbitos intra-hospitalares e possibilidade de revascularização concomitante do miocárdio. Dentre os pacientes estudados, muitos foram acompanhados por ecocardiografia e ventriculografia, porém esses métodos não foram considerados neste estudo, pois as técnicas imputadas não foram específicas para avaliação correta da função ventricular. A sobrevivência tardia foi avaliada por curvas atuariais de sobrevivência de Kaplan-Meier, sendo destas excluídos os óbitos intra-hospitalares e os pacientes que foram perdidos durante o intervalo do estudo. As diferenças estatísticas ( $p$ valor) foram medidas pelos métodos de "Log Rank" nas curvas atuariais de sobrevivência e pelo método de "Nonparametric Test" (teste de duas simples proporções) nas demais comparações. A probabilidade de rejeição foi de $95 \%$. O intervalo de pesquisa do estudo foi de quinze anos.

\section{Técnica Operatória}

Todos os procedimentos cirúrgicos foram realizados por esternotomia mediana e circulação extracorpórea (CEC), com canulação da aorta ascendente e das veias cavas ou átrio direito. Nos primeiros casos foi utilizada hipotermia moderada $\left(28^{\circ} \mathrm{C}\right)$ e cardioplegia cristalóide, passando, em seguida, para CEC isotérmica $\left( \pm 32^{\circ} \mathrm{C}\right) \mathrm{com}$ cardioplegia sangüínea, isotérmica, com proporção sangue/solução de 4:1, anterógrada (raiz da aorta) e/ou retrógrada (seio coronário).

Para reparo linear (Figura 1), o aneurisma foi ressecado, o trombo mural removido, quando presente, e as bordas foram suturadas utilizando duas tiras de patch de pericárdio bovino ou teflon para apoio, com fio de poliéster 2.0 em sutura contínua. Na reconstrução geométrica de VE (Figura 2), foi ressecada amplamente a área necrótica do aneurisma, e com um medidor apropriado, foi avaliado o diâmetro da prótese (anel semi-rígido de pericárdio bovino) a ser utilizado, conforme proposto por BRAILE et al. (8). Com fios de poliéster 2.0, foram passados pontos em "U" nas bordas do colo aneurismático e na prótese e, em seguida, posicionando-a e fixando-a com múltiplos nós.

Após correção do aneurisma, foi feita a revascularização miocárdica, quando necessária ou quando possível, utilizando enxertos de artéria torácica interna e/ou de veia safena. 
Sgarbi C J, Ardito R V, Santos R C, Bogdan R A B, Arruda Junior F V, Silva E M, Benites A C M, Ardito W R, Neves M F F B - Correção cirúrgica de aneurisma de ventrículo esquerdo: comparação entre as técnicas de sutura linear e reconstrução geométrica. Rev Bras Cir Cardiovasc 2000; 15(4): 293-301.

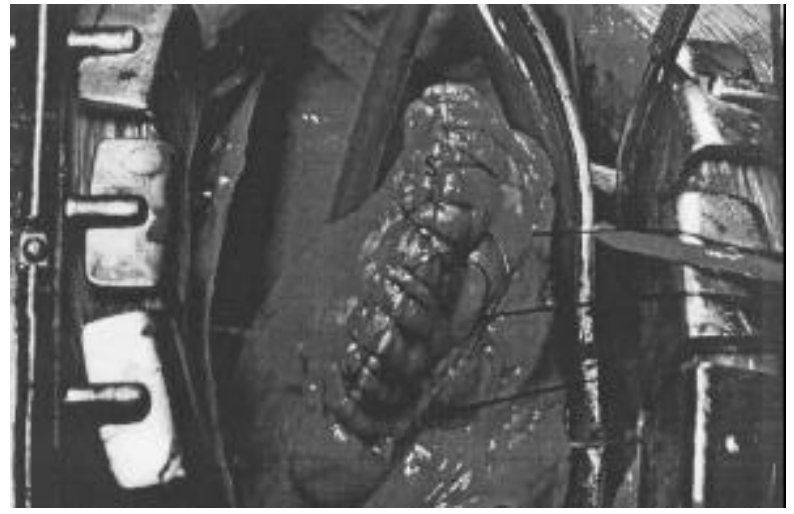

Fig. 1 - Correção do aneurisma de VE (apical) com utilização do método de sutura $(\mathrm{SL})$

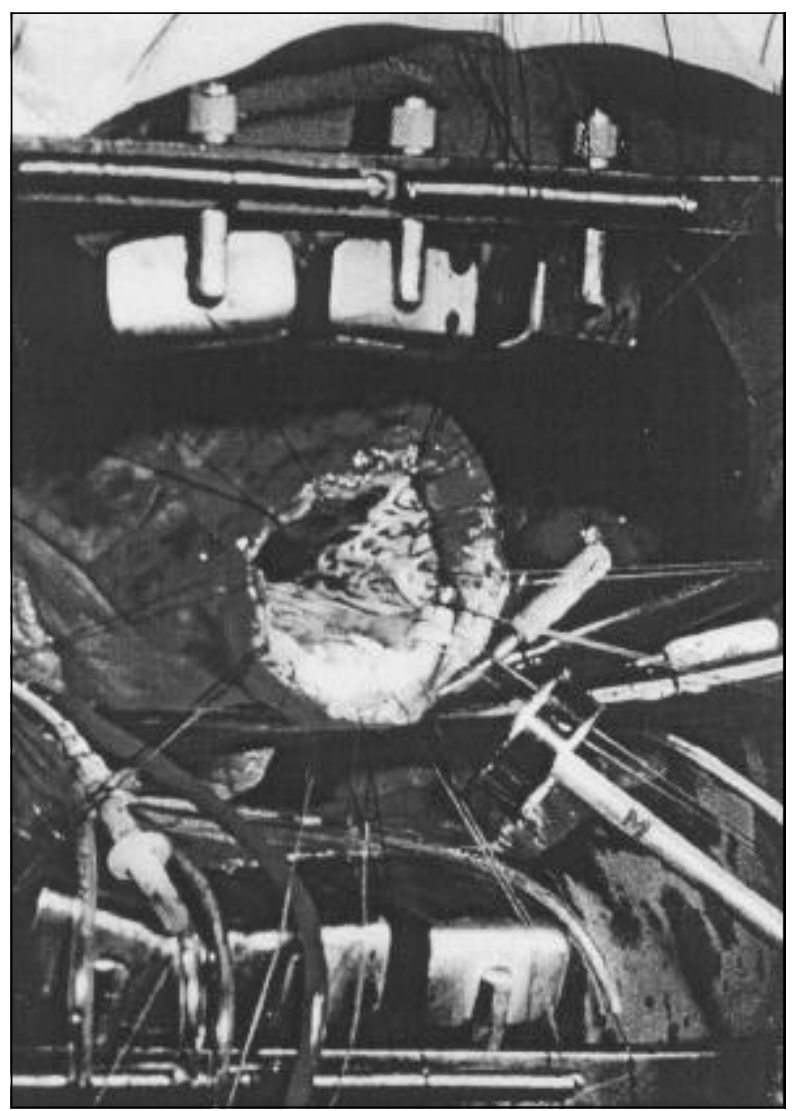

Fig. 2 - Correção do aneurisma de VE (apical) com utilização do método de reconstrução. $M$ = medidor de diâmetro para implante do anel semi-rígido.

\section{RESULTADOS}

O estudo da curva atuarial no acompanhamento dos pacientes submetidos a operação para correção do aneurisma de VE, pelas técnicas de sutura linear e reconstrução geométrica, revelou uma sobrevi-

\section{GRÁFICO 1}

CURVA ATUARIAL DE SOBREVIVÊNCIA DOS PACIENTES SUBMETIDOS A SUTURA LINEAR E RECONSTRUÇÃO GEOMETRICA DO VENTRÍCULO ESQUERDO

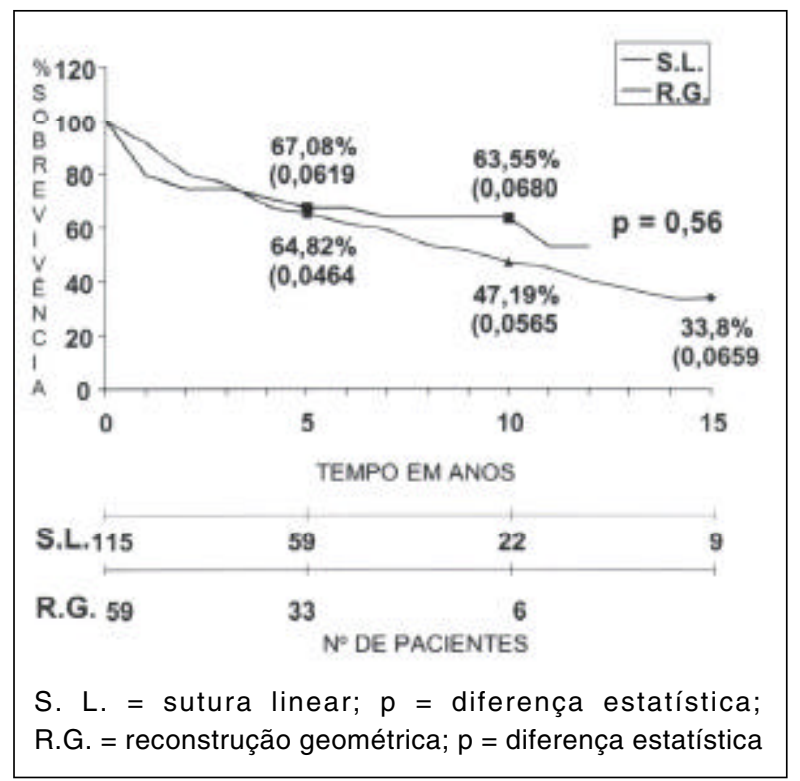

GRÁFICO 2

CURVA ATUARIAL DE SOBREVIVÊNCIA DOS DOIS GRUPOS ASSOCIADOS (SUTURA LINEAR E RECONSTRUÇÃO GEOMÉTRICA)

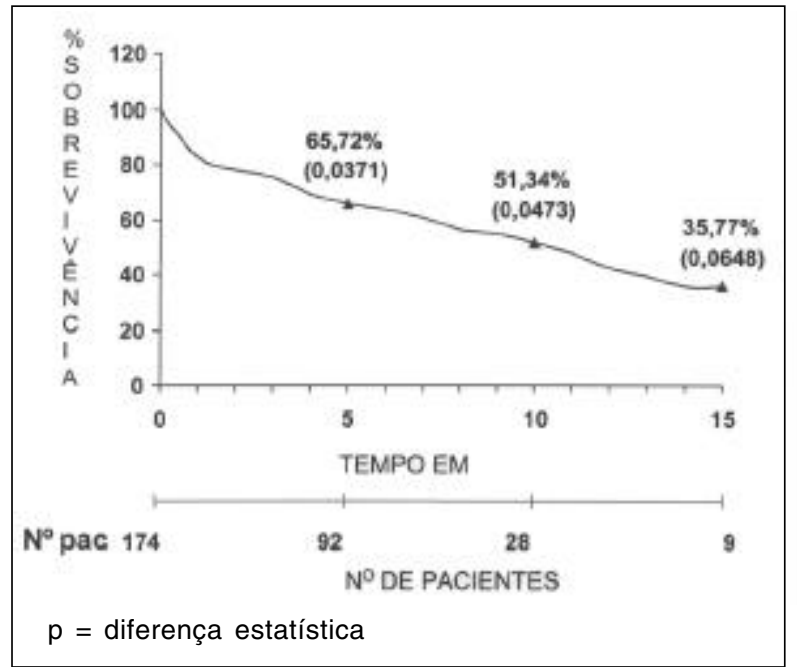

vência em 10 anos de 47,19\% (EP=0,0565) nos pacientes do primeiro grupo e de 63,55\% $(E P=0,0680)$ nos do segundo grupo (Gráfico 1 ), não havendo diferença significativa entre as duas técnicas $(p=0,56)$. A sobrevivência média, considerados os dois grupos associados, foi de $51,34 \%$ 
Sgarbi C J, Ardito R V, Santos R C, Bogdan R A B, Arruda Junior F V, Silva E M, Benites A C M, Ardito W R, Neves M F F B - Correção cirúrgica de aneurisma de ventrículo esquerdo: comparação entre as técnicas de sutura linear e reconstrução geométrica. Rev Bras Cir Cardiovasc 2000; 15(4): 293-301.

TABELA 1

\begin{tabular}{lcc}
\hline & CAUSAS DE ÓBITO INTRA-HOSPITALAR & \\
\hline \multicolumn{1}{c}{ CAUSA } & $\begin{array}{c}\text { SUTURA } \\
\text { LINEAR }\end{array}$ & $\begin{array}{c}\text { RECONSTRUÇÃO } \\
\text { GEOMÉTRICA }\end{array}$ \\
\hline Hemorragia & 0 & 1 \\
\hline Septicemia & 1 & 2 \\
\hline Embolia cerebral & 0 & 1 \\
Arritmia & 4 & 0 \\
Choque cardiogênico & 3 & 4 \\
Intra-operatório & 1 & 0 \\
\hline Insuficiência renal & 0 & 1 \\
\hline Embolia pulmonar & 1 & 0 \\
Ignorado & 4 & 2 \\
TOTAL & 14 & 11 \\
\hline
\end{tabular}

$(E P=0,0473)$ em 10 anos e de $35,77 \%(E P=0,0648)$ em 15 anos (Gráfico 2).

A ocorrência de óbito intra-hospitalar foi de 14 casos no método de sutura linear, perfazendo um total de $9,65 \%$ de todos os pacientes submetidos a esta técnica, e de 11 casos no método de reconstrução geométrica, num total de $16,18 \%$; não tendo, portanto, diferença estatística $(p=0,17)$. As causas dos óbitos estão na Tabela 1.

Quanto a presença e retirada de trombo na cavidade ventricular esquerda, dos pacientes operados pelo método de sutura linear, 46 (31,72\%) pacientes foram submetidos a tal procedimento e, dos operados pelo método de reconstrução geométrica, $30(44,12 \%)$ pacientes necessitaram também da retirada do trombo do $\mathrm{VE}$, não demonstrando nenhuma diferença estatística $(p=0,07)$.

A revascularização do miocárdio foi realizada sempre que necessário, quando factível, e visando a revascularização mais completa possível. Dos 145 pacientes submetidos a sutura linear, 100 (69\%) pacientes receberam enxertos em coronárias e 47 (31\%) pacientes não foram revascularizados. A mediana de pontes neste grupo foi de 2 pontes por paciente. A coronária interventricular anterior (IA) foi revascularizada em 71 pacientes $(71 \%$ dos revascularizados e $49 \%$ do total dos pacientes) e a artéria torácica interna esquerda e/ou direita foi utilizada em 33 (33\%) pacientes. Na reconstrução geométrica do VE, do total de 68 pacientes, 58 $(85,3 \%)$ foram revascularizados e somente 8 $(13,8 \%)$ não receberam pontes. A mediana de pontes nestes pacientes também foi de 2 pontes por paciente. A IA foi revascularizada em 40 doentes $(69 \%$ dos revascularizados e em $61 \%$ do total) e a artéria torácica interna utilizada em 20

\section{GRÁFICO 3}

CURVA ATUARIAL DE SOBREVIVÊNCIA DOS PACIENTES REVASCULARIZADOS X NÃO REVASCULARIZADOS

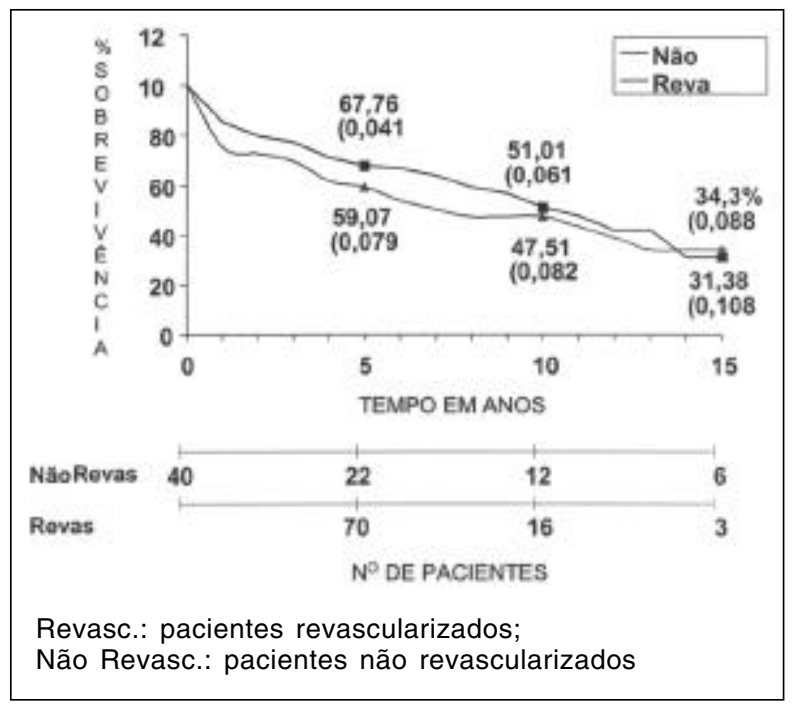

(34\%). A análise associada da sobrevivência de todos os pacientes operados, comparando revascularizados com os não revascularizados, mostrou que, no intervalo de 0 a 5 anos do estudo, não houve diferença estatística entre esses grupos ( $p=0,05)$; portanto, analisando o intervalo de 0 a 10 anos, houve diferença significativa quanto à sobrevivência $(p=0,008)$, demonstrando a melhor evolução dos pacientes revascularizados. No intervalo de 0 a 15 anos, também não foi demonstrada diferença estatística na sobrevivência dos dois grupos $(p=0,05)$. 
Sgarbi C J, Ardito R V, Santos R C, Bogdan R A B, Arruda Junior F V, Silva E M, Benites A C M, Ardito W R, Neves M F F B - Correção cirúrgica de aneurisma de ventrículo esquerdo: comparação entre as técnicas de sutura linear e reconstrução geométrica. Rev Bras Cir Cardiovasc 2000; 15(4): 293-301.

TABELA 2

\begin{tabular}{lccccccc}
\hline \multicolumn{1}{c}{} & \multicolumn{1}{c}{ RESULTADOS } \\
\hline & $\begin{array}{c}\text { Sutura } \\
\text { Linear }\end{array}$ & $\begin{array}{c}\text { \%SL } \\
\text { Geométrica }\end{array}$ & Rec. & \%R.G. & Total & \%Total & p Valor \\
\hline O.I.H & 14 & $9,65 \%$ & 11 & $16,18 \%$ & 25 & $11,70 \%$ & 0,17 \\
R.T.VE & 46 & $31,72 \%$ & 30 & $44,12 \%$ & 76 & $35,70 \%$ & 0,07 \\
R.M. & 100 & $68,96 \%$ & 58 & $85,29 \%$ & 158 & $74,18 \%$ & 0,014 \\
\hline
\end{tabular}

O.I.H.: Óbitos intra-hospitalares; R.T.VE: retirada de trombo do ventrículo esquerdo; R.M.: revascularização do miocárdio; \%S.L. porcentagem de pacientes submetidos a sutura linear; \% R.G.: porcentagem de pacientes submetidos a reconstrução geométrica do VE; \% Total: porcentagem do total dos pacientes somados os dois grupos; $p$ Valor: diferença estatística; Colunas 2, 4 e 6: números de pacientes.

A Tabela 2 resume os principais dados dos resultados encontrados no estudo, com exceção das curvas atuariais.

\section{COMENTÁRIOS}

O primeiro sucesso cirúrgico da operação de correção do aneurisma de ventrículo esquerdo foi reportado por COOLEY et al. ${ }^{(5)}$, em 1958, utilizando o método de sutura linear. Esse método foi tido como técnica de escolha pela maioria dos cirurgiões, pela simplicidade e pela eficiência. Em 1979, LEVINSKY et al. (9) relataram a reconstrução geométrica de VE com o uso de patch de dacron, após ressecção de um largo aneurisma de VE JATENE (1), sugeriu que o método de sutura linear poderia distorcer a geometria ventricular, alterando a sua função, com conseqüente diminuição do volume ventricular, criando, portanto, o conceito de reconstrução geométrica do VE.

Em nossos pacientes, o método de reconstrução geométrica do VE empregado, na grande maioria das vezes, foi a ressecção ampla da área aneurismática e o implante de um anel semi-rígido de pericárdio bovino, desenvolvido por BRAILE et al. ${ }^{(8)}$.

Vários estudos comparativos entre os métodos de sutura linear e reconstrução geométrica do VE apontam o segundo como o de superior resultado clínico, como os trabalhos de VICOL et al. (10) que, em um acompanhamento de 10 anos, após correção cirúrgica do aneurisma de VE, empregando também as duas técnicas, verificaram melhor função ventricular no grupo submetido a reconstrução geométrica do VE, apesar dos índices de mortalidade tardia serem semelhantes nos dois grupos; VURAL et al. (11), que também não encontraram diferença estatística quanto à sobrevivência entre os dois grupos acompanhados, demonstrando vantagens quanto à função ventricular para os pacientes submetidos a recons- trução geométrica do VE; SINATRA et al. (12), que obtiveram como resultado melhor classe funcional a longo prazo, nos pacientes submetidos a reconstrução geométrica do VE em relação aos submetidos a sutura linear, sem nenhuma diferença quanto à sobrevivência; SHAPIRA et al. (13), também relatando vantagens, somente quanto a função ventricular, na reconstrução geométrica.

Em nossos pacientes estudados, não encontramos diferença entre os dois métodos cirúrgicos empregados, quanto a curva de sobrevivência, mortalidade intra-hospitalar e retirada de trombo da cavidade ventricular esquerda; no entanto, a função cardíaca, a evolução clínica, a classe funcional e os dados ecocardiográficos não foram possíveis de serem analisados, havendo, portanto, necessidade de dados mais conclusivos. Cabe aqui citar que, apesar de tudo, houve uma tendência à obtenção de melhores resultados nos pacientes submetidos a reconstrução geométrica.

Outros estudos comparativos publicam relatos compatíveis com o nosso, como o trabalho de KESLER et al. (14), onde estes não encontraram nenhuma diferença estatística entre os dois grupos estudados, quanto aos parâmetros ecocardiográficos, classe funcional e sobrevivência; KAWATA et al. (15) relataram resultados semelhantes ao anterior, porém com um curto período de acompanhamento.

Apesar dos dados importantes encontrados em todos os estudos comparativos citados, incluindo o nosso, estes carecem de melhores detalhes e dados mais específicos para obtenção de maiores conclusões. Outra observação importante é: será que os pacientes piores não são aqueles que vão para reconstrução geométrica do VE? Em nosso estudo não foi possível avaliar este item com precisão, porém, por haver, em uma grande porção dos pacientes estudados, uma seqüência na utilização do método de sutura linear, seguido de reconstrução geométrica do VE, não houve, pelo menos 
Sgarbi C J, Ardito R V, Santos R C, Bogdan R A B, Arruda Junior F V, Silva E M, Benites A C M, Ardito W R, Neves M F F B - Correção cirúrgica de aneurisma de ventrículo esquerdo: comparação entre as técnicas de sutura linear e reconstrução geométrica. Rev Bras Cir Cardiovasc 2000; 15(4): 293-301.

nestes, uma escolha específica para cada caso isoladamente.

A revascularização do miocárdio teve uma participação positiva e significativa na curva de sobrevivência dos nossos pacientes operados para correção do aneurisma de VE. BACIEWICZ et al. (16) relataram resultados semelhantes quanto à sobrevivência, em um estudo com 10 anos de acompanhamento. DOR et al. (17) demonstraram melhora significativa da função ventricular nos pacientes submetidos a correção do aneurisma de VE associado com revascularização do miocárdio. Também fica a dúvida se a revascularização do miocárdio não foi realizada com mais freqüência nos pacientes que se apresentavam em melhor estado clínico. Porém, apesar disto, esses dados são significativos e servem de parâmetro para que se faça, nestes pacientes, a revascularização do miocárdio mais completa possível.

A mortalidade intra-hospitalar foi alta em nosso estudo, porém os índices encontrados são semelhantes aos da literatura, como, por exemplo, os estudos de SINATRA et al. (12) e de VICOL et al. (10). Também é importante ressaltar que o alto índice de mortalidade encontrado em nosso estudo se deve, principalmente, ao fato de ser este de longa data, sendo que, atualmente, com a evolução tecnológica e, principalmente, com a melhora dos métodos de proteção miocárdica, este índice caiu significativamente.

Por não haver nenhuma diferença entre os grupos quanto ao método de proteção miocárdica, extracorpórea e grau de revascularização miocárdica, nós acreditamos que, apesar de tudo, os resultados comparativos entre os grupos são válidos.

\section{CONCLUSÃO}

O presente estudo demonstrou curvas de sobrevivência semelhantes entre os pacientes submetidos às técnicas de sutura linear e reconstrução geométrica do VE. A incidência de mortalidade intra-hospitalar foi alta, porém compatível com índices encontrados na literatura. Entre a totalidade dos pacientes operados, também se demonstrou a superioridade na evolução dos pacientes revascularizados sobre os não revascularizados.

RBCCV 44205-515

Sgarbi C J, Ardito R V, Santos R C, Bogdan R A B, Arruda Junior F V, Silva E M, Benites A C M, Ardito W R, Neves M F F B - Surgical correction of left ventricular aneurysm: comparison between linear suture and geometric reconstruction techniques. Rev Bras Cir Cardiovasc 2000; 15(4): 293-301.

ABSTRACT: Objective: To evaluate the evolution up to 15 years of patients who underwent surgical correction of left ventricular aneurysm and to compare the techniques of linear suture and geometric reconstruction.

Methods: We studied 213 patients, of which 166 (77.9\%) were men. The mean age was 53.1 years $(S D=9.9$ years). Of these, $145(68 \%)$ underwent repair using the linear suture technique and $68(32 \%)$ underwent the geometric reconstruction technique. The Kaplan - Meier method was used to evaluate late survival. We evaluated the presence of mural thrombus, in and out of hospital mortality and the possibility of myocardial revascularization in the same procedure. Statistical differences were measured using the Log Rank method for actuarial curves and the nonparametric test in the remaining data compared.

Results: Our survival curves after a follow up of 10 years demonstrated that patients submitted to the linear suture and LV geometric reconstruction had a survival of $47.19 \%(S E M=0.056)$ and $63,55 \%(S E M=0.068)$, respectively, which were not statistically significantly different $(p=0.56)$. The overall survival in the 2 groups together was $51.34 \%(\mathrm{SEM}=0.0473)$ at 10 years and $35,77 \%(\mathrm{SEM}=0.0684)$ at 15 years. The in hospital death was $9.5 \%$ for the linear suture and $16.6 \%$ for the geometric reconstruction $(p=0.17)$. The removal of LV thrombus was $31.29 \%$ and $45.45 \%$, respectively $(p=0.07$ ). Revascularization was performed in, $69 \%$ of the patients in the linear suture group and $85.3 \%$ in the geometric reconstruction group. Comparing patients who were revascularized with those who were not, there was a better survival at 10 years for the first group $(p=0.008)$.

Conclusions: There was no statistically significant difference in the survival of patients who underwent linear suture compared to geometric reconstruction of the LV for aneurysm repair. Our results demonstrated superior survival in those patients who could undergo revascularization in the same procedure.

DESCRIPTORS: Heart ventricle, surgery. Heart aneurysms, surgery. Heart ventricle aneurysm, surgery, comparative study. Heart ventricle aneurysm, surgery, methods. Surgical anastomoses, methods. 
Sgarbi C J, Ardito R V, Santos R C, Bogdan R A B, Arruda Junior F V, Silva E M, Benites A C M, Ardito W R, Neves M F F B - Correção cirúrgica de aneurisma de ventrículo esquerdo: comparação entre as técnicas de sutura linear e reconstrução geométrica. Rev Bras Cir Cardiovasc 2000; 15(4): 293-301.

\section{REFERÊNCIAS BIBLIOGRÁFICAS}

1 Jatene A D - Left ventricular aneurysmectomy: resection or reconstruction. $J$ Thorac Cardiovasc Surg 1985; 89: 321-31.

2 Calbet J M, Obi C, Rodriguez R et al. - Aneurysms of the left ventricle: surgical treatment. Rev Esp Cardiol 1998; 51(suppl 3): 80-5.

3 Abrams D L, Edelist A, Luria M H, Miller A J Ventricular aneurysm: a reappraisal on a study of 65 consecutive autopsied cases. Circulation 1963; 27: 164 .

4 Likoff W \& Bailey C P - Ventriculoplasty: excision of myocardial aneurysm: report of a successful case. JAMA 1958; 915-20.

5 Cooley D A, Collins H A, Morris G C, Chapman D W - Ventricular aneurysm after myocardial infarction: surgical excision with use of temporary cardiopulmonary bypass. JAMA 1958; 167: 557-60.

6 Dor V, Saab M, Coste P, Kornaszewska M, Montiglio F - Left ventricular aneurysm: a new surgical approach. Thorac Cardiovasc Surg 1989; 37: 11-9.

7 Cooley D A - Repair of pos-infarction aneurysm of the left ventricle. In: Cooley D A, ed. Cardiac surgery: state of the art reviews. Philadelphia: Handley and Belfus, 1990: vol 4 (2) p.309.

8 Braile D M, Mustafá R M, Santos J L V et al. Correção da geometria de ventrículo esquerdo com prótese semi-rígida de pericárdio bovino. Rev Bras Cir Cardirvasc 1991; 6: 109-15.

9 Levinsky L, Arani D T, Raza S T, Kohn R, Schimert $G$ - Dacron patch enlargement of anterior wall of left ventricle after aneurysmectomy with concomitant infarctectomy. J Thorac Cardiovasc Surg 1978; 77: 753-6.

10 Vicol C, Rupp G, Fischer S, Summer C, Dietrich B $H$, Struck E - Linear repair versus ventricular reconstruction for treatment of left ventricular aneurysm: a 10-year experience. J Cardiovasc Surg (Torino) 1998; 39: 461-7.

11 Vural K M, Sener E, Ozatik M A, Tasdemir O, Bayazit K - Left ventricular aneurysm repair: an assessment of surgical treatment modalities. Eur J Cardiothorac Surg 1998; 13: 49-56.

12 Sinatra R, Macrina F, Braccio $M$ et al. - Left ventricular aneurysmectomy; comparison between two techniques; early and late results. Eur J Cardiothorac Surg 1997; 12: 291-7.

13 Shapira O M, Davidoff R, Hilkert R J, Aldea G S, Fitzgerald C A, Shemin R J - Repair of left ventricular aneurysm: long-term results of linear repair versus endoaneurysmorrhaphy. Ann Thorac Surg 1997; 63: 701-5.

14 Kesler K A, Fiore A C, Naunheim K S et al. - Anterior wall left ventricular aneurysm repair: a comparison of linear versus circular closure. J Thorac Cardiovasc Surg 1992; 103: 841-8.

15 Kawata T, Kitamura S, Kawachi K et al. - Cardiac function following left ventricular aneurysm repair: comparison between patch reconstruction and direct closure methods. Nippon Kyobu Geka Gakkai Zasshi. 1993; 41: $2075-80$.

16 Baciewicz P A, Weintraub W S, Jones E L et al. - Late follow-up after repair of left ventricular aneurysm and (usually) associated coronary bypass grafting. $A m \mathrm{~J}$ Cardiol 1991; 68: 193-200.

17 Dor V, Sabatier M, Di Donato M, Maioli M, Toso A, Montiglio F - Late hemodynamic results after left ventricular patch repair associated with coronary grafting in patients with postinfarction akinetic on dyskinetic aneurysm of the left ventricle. J Thorac Cardiovasc Surg 1995; 110: 1291-301.

\section{Discussão (transcrições de fita gravada)}

\author{
DR. RICARDO NILSSON SGARBIERI \\ São Paulo, SP
}

Gostaria de agradecer à Comissão Organizadora do Congresso pelo convite para comentar este trabalho. Poucos são os trabalhos na literatura que comparam as técnicas de sutura linear e reconstrução geométrica. Dr. Adib Jatene, em 1985, quando introduziu o conceito de reconstrução geométrica, demonstrou que na sua experiência a adoção desta estratégia levou a uma redução na mortalidade de $11,6 \%$ para $4,3 \%$, e também da mortalidade tardia de $12,5 \%$ para $3 \%$. Resultados semelhantes foram demonstrados por Dor e colaboradores, em 1989, quando comparam seus primeiros 100 casos de reconstrução geométrica com 40 casos de sutura linear do mesmo Serviço. Demonstraram, além de redução da mortalidade hospitalar, uma melhora significativa da função sistólica do ventrículo esquerdo após a reconstrução geométrica. Cooley e colaboradores, em 1990, quando propuseram a endoaneurismorrafia, compararam controles históricos de seu Serviço submetidos a aneurismectomia clássica com sutura linear com pacientes submetidos a endoaneurismorrafia. Também encontraram melhora sintomática da função ventricular em uma proporção significativamente maior nos pacientes submetidos à reconstrução geométrica. O título do presente trabalho nos leva a crer que também se trata de uma comparação entre as duas técnicas, no entanto, nos ficou uma dúvida se os 2 grupos 
Sgarbi C J, Ardito R V, Santos R C, Bogdan R A B, Arruda Junior F V, Silva E M, Benites A C M, Ardito W R, Neves M F F B - Correção cirúrgica de aneurisma de ventrículo esquerdo: comparação entre as técnicas de sutura linear e reconstrução geométrica. Rev Bras Cir Cardiovasc 2000; 15(4): 293-301.

de pacientes são comparáveis quanto aos parâmetros pré-operatórios. Não encontramos no trabalho nenhuma tabela ou descrição das características pré-operatórias dos 2 grupos que nos permitissem esta conclusão. Os autores em sua Discussão e na sua apresentação questionam se os pacientes piores não são aqueles que vão para reconstrução geométrica? Isso poderia explicar o fato de que a sobrevivência, em 10 anos, apesar de ter sido maior no grupo da reconstrução geométrica, essa diferença não apresentou significância estatística. Outro fato que pode ter influenciado nesta conclusão e que também não ficou claro no texto do trabalho é qual foi a porcentagem de pacientes perdidos ao longo do seguimento de ambos os grupos. A segunda conclusão a que os autores chegaram se refere à revascularização concomitante do miocárdio, associada à correção cirúrgica do aneurisma de ventrículo esquerdo. Os autores demostraram, de modo elegante, que a revascularização associada melhora a sobrevida a longo prazo. Esse fato foi inicialmente demonstrado por Boliac, em 1984, e já foi reforçado na literatura por vários trabalhos, como os próprios autores discutem no presente artigo. Nos chamou a atenção a freqüência relativamente baixa de emprego da artéria torácica interna na revascularização, apenas 33\% no grupo de sutura linear e $34 \%$ no grupo de reconstrução geométrica. Gostaria de saber dos autores se há alguma explicação para este fato na presente casuística. Gostaria ainda de saber qual é a técnica preferencialmente empregada pelo Serviço atualmente, apesar de não haver diferença na sobrevivência a longo prazo. A conclusão do trabalho foi pertinente, coerente com os dados apresentados, fato pelo qual parabenizo os autores, não fazendo inferências a partir dos seus dados. Entretanto, gostaria de chamar a atenção dos colegas para o fato de que a conclusão do trabalho é específica apenas quanto à diferença estatística sobre sobrevivência a longo prazo entre 2 grupos ou 2 técnicas estudadas. Isto não significa, entretanto, que os resultados das técnicas em questão, sutura linear e reconstrução geométrica, são iguais ou mesmo comparáveis. A análise é apenas da mortalidade e, consequentemente, da sobrevivência, não nos parece suficientemente apurada para avaliar o resultado de uma técnica cirúrgica na correção de uma doença que é multifatorial como aneurisma do ventrículo esquerdo. Deste modo, fica aqui uma palavra de cautela de que devemos observar com cuidado qual é o resultado final que buscamos com a correção do aneurisma do ventrículo esquerdo, se é a melhora mantida a longo prazo dos sintomas de insuficiência cardíaca devemos procurar parâmetros mais sensíveis de função ventricular esquerda para compararmos estas técnicas distintas.

\section{DR. JARBAS J. DINKHUYSEN}

São Paulo, SP

O que importa não é o tamanho do aneurisma o que importa, na realidade, é a porção de ventrículo que vai manter o débito depois da correção. Muitas vezes, a fração de ejeção é alta, mas a porção residual de músculo útil é muito boa, fazendo com que a evolução a longo prazo seja satisfatória, independentemente do próprio tipo de correção. Acho que o trabalho precisaria estratificar, pré-operatoriamente, aqueles pacientes que têm uma função contrátil residual boa e aqueles que esta é ruim, isto é, aqueles pacientes que têm um colo formado e depois forma-se o aneurisma, diferentemente daqueles pacientes que têm já um colo aberto e com uma fração de ejeção muito baixa das porções residuais. Outro aspecto que deixou dúvida foi a comparação entre os pacientes revascularizados e os não-revascularizados, fazendo pressupor que os doentes revascularizados teriam melhor resultado. Não entendi bem porque os outros doentes não foram revascularizados, se eles não foram revascularizados, na minha opinião, é porque não havia indicação, motivo pelo qual não há uma razão para fazer uma comparação entre um e outro grupo. Acho que um dos resultados básicos na correção do aneurisma do ventrículo esquerdo é a revascularização subjacente. O trabalho ficaria muito bom se fosse feita a comparação, única e exclusivamente, do tratamento do aneurisma sem os procedimentos adicionais, assim, definitivamente teríamos uma noção bem clara, levando em conta também a análise da fração de ejeção da porção residual útil que fica próxima à região do aneurisma.

\section{DR. ADIB D. JATENE}

São Paulo, SP

São procedimentos completamente diferentes, se a obstrução é antes da primeira septal é um problema, se, ao contrário, é depois, o problema é outro. É difícil comparar aneurismas sem saber de que tipo está se falando. Outro aspecto importante é o grau de comprometimento do septo, e como este foi tratado ou não. Quanto ao conceito de correção geométrica, tenho a impressão que quando se fala em correção geométrica quer dizer que foi colocado um remendo. Não é isto; correção da geometria significa restabelecer a forma do ventrículo normal e, freqüentemente, é preciso tracionar a borda da parede livre do ventrículo esquerdo e, com isso, o músculo papilar. Assim, a correção da geometria, não é simplesmente colocar uma placa, ela deve ser colocada, mas depende da forma como isto é feito. Continuo insistindo que se a cirurgia for conduzida adequadamente a mortalidade deveria ser zero. Acontece que, por 
Sgarbi C J, Ardito R V, Santos R C, Bogdan R A B, Arruda Junior F V, Silva E M, Benites A C M, Ardito W R, Neves M F F B - Correção cirúrgica de aneurisma de ventrículo esquerdo: comparação entre as técnicas de sutura linear e reconstrução geométrica. Rev Bras Cir Cardiovasc 2000; 15(4): 293-301.

mais que a gente trabalhe, não conseguimos corrigir adequadamente. Em vários casos, tive que desmanchar a correção e fazer outra para manter baixa a pressão do átrio esquerdo e etc. Portanto, corrigir a geometria do ventrículo esquerdo não é uma tarefa simples, é muito complicado. A mortalidade é apenas um dos fatores, mas o que é fundamental é a situação clínica no pós-operatório tardio, porque o que mais preocupava nas experiências publicadas na literatura, no passado, com sutura linear é que o doente não morria, mas ele também não melhorava. Desta maneira, tem uma série de aspectos que precisam ser considerados e, principalmente, homogeneizar grupos e comparar o que é comparável, pois os aneurismas nem sempre são comparáveis, o comprometimento anatômico é muito diferente de caso para caso. Eu sempre insisto que se analise cuidadosamente 0 padrão arterial; uma coisa é uma artéria descendente anterior que tem duas ou três diagonais e que a obstrução compromete as diagonais, outra coisa é uma descendente anterior que praticamente não tem diagonais, não estando comprometidas, havendo a presença do ramo diagonalis. Isto muda, completamente, o comprometimento do músculo que foi infartado e o contingente de septo que está comprometido. É necessário ver como são as artérias que vêm da descendente posterior e da descendente anterior, para quantificar o comprometimento do septo, tudo isto tem que ser considerado na correção do aneurisma. A correção da geometria do ventrículo esquerdo pode ser feita com sutura linear ou com plicatura, não precisa ser remendo.
O conceito precisa ser revisto, pois a idéia que eu tenho quando eu assisto as apresentações é que correção geométrica é quando põe o remendo, que pode ser colocado torto, no sentido vertical ou horizontal, mas é correção geométrica.

\section{DR. SGARBI \\ (Encerrando)}

Respondendo algumas perguntas do Dr. Ricardo, não foi possível selecionar os pacientes no período pré-operatório, já que trata-se de uma pesquisa que abrange um grande período, de pacientes operados desde 1973. Quanto à utilização da artéria torácica interna ser de $36 \%$, ou seja, ser baixa, isto se deve ao mesmo fato, já que o estudo engloba pacientes desde 1973, e naquela época, usava-se muito menos este tipo de enxerto. Atualmente, estamos utilizando a artéria torácica interna em quase todos os pacientes. Em relação a preferência do método utilizado é um fato muito interessante. Em um período inicial, de 1973 a 1985, foi utilizada a sutura linear em quase todos os pacientes, de 1985 até meados da década de 90 , houve uma preferência pela técnica reconstrução geométrica e de 1990 até os dias atuais é que há uma escolha pelo um método específico. O método que utilizamos atualmente para reconstrução geométrica é o método tipo Braile, com o implante de um anel semi-rígido por ser uma prótese inelástica em aneurismas maiores. Em aneurismas menores, utilizamos a técnica simples de sutura linear. 